\title{
Rotavirus Infection in Children and Adult Patients Attending in a Tertiary Hospital of São Paulo, Brazil
}

\author{
Emerson Carraro ${ }^{1,2}$, Ana Helena Sitta Perosa ${ }^{2}$, Itacy Siqueira ${ }^{2}$, Jacyr Pasternak ${ }^{2}$ and Marines Dalla Vale Martino ${ }^{2}$ \\ ${ }^{I}$ Clinical Virology Laboratory, Infectious Diseases Department, UNIFESP; ${ }^{2}$ Microbiology Section, Clinical Laboratory, Albert Einstein Hospital; \\ São Paulo, SP, Brazil
}

\begin{abstract}
During the period of January 2003 to December 2005, 3,768 stool samples were received in the Microbiology Laboratory for rotavirus antigen detection from outpatients and inpatients of Albert Einstein Hospital, SP. Fresh stool samples from children and adults were analyzed by two methodologies: during 2003 and 2004 by latex agglutination (Slidex Rotavirus, Biomerieux) and 2005 by an immunochromatographic assay for the combined detection of rotavirus and adenovirus (Vikia Rota-Adeno, Biomerieux). Rotavirus group A was detected in 755 (20\%) samples. The annual prevalence was $19.8 \%$ in $2003,21.7 \%$ in 2004, and $18.7 \%$ in 2005 . Rotavirus was detected every month during the period of the study, with peak of positivity between June and August ( $>35 \%)$. The prevalence in hospitalized patients was $26.1 \%(352 / 1,350)$ and in outpatients was $16.7 \%(403 / 2,418)$. For hospitalized patients most of the rotavirus infections were diagnosed in Pediatric setting, age range of 0 to 10 years (prevalence of 55.3\%, 295/ 534). Overall positivity was up to $30 \%$ in patients between six months and five years of age $(67 \%$ of all positive patients), all other age groups had at least $10 \%$ positive tests. Rotavirus infection is common in Sao Paulo, and besides the expected higher frequency in children it is also frequent in adults.

Key-Words: Rotavirus, gastroenteritis.
\end{abstract}

Rotavirus is the most common viral infection leading to acute diarrhea in children worldwide [1,2]. Previous review showed that rotavirus infection is responsible for $22 \%$ of all hospitalization due to acute diarrhea in children, and 600,000 or even more deaths caused by diarrhea in children aged less than five years are caused by rotavirus infection [3].

Many studies done in Brazil documented the relevance of rotavirus infection as a cause of severe diarrhea among young children. The estimated mortality is 2,500 deaths per year [4]. Rotavirus vaccine was introduced in the vaccination routine in Brazil in 2006, and in the next years it will be possible to evaluate the impact of this vaccination in all ages [5]. Few studies address to rotavirus associated gastroenteritis in adults. Some reports described rotavirus gastroenteritis in adults in close contact with children, as family contacts [6], day care nursery [7], small rural city [8] and patients attending private care hospital [9].

This study describes epidemiological aspects of rotavirusassociated gastroenteritis in hospitalized and outpatients, children and adults, in a Brazilian tertiary care hospital in Sao Paulo, Brazil.

\section{Material and Methods}

A retrospective study was done analyzing all rapid tests to rotavirus obtained in the Microbiology Laboratory of Hospital Albert Einstein, from January 2003 to December 2005. Albert Einstein Hospital is a tertiary care Hospital that deals with a high socioeconomic level population in

Received on 15 September 2007; revised 11 January 2008.

Address for correspondence: Dr. Emerson Carraro. Laboratório de Virologia Clínica. Rua Pedro de Toledo, 781, andar 15. Vila Clementino, São Paulo - SP, Brasil. Zip code: 04039-032. Fone/fax: 55(11)50815394.E-mail: emersoncarraro@bol.com.br.

The Brazilian Journal of Infectious Diseases 2008;12(1):44-46. (C) 2008 by The Brazilian Journal of Infectious Diseases and Contexto Publishing. All rights reserved. it's emergency room and admitted patients, but besides that cares for a slum population as outpatients only. Two methods were used to detect rotavirus antigen on fresh stool samples: from 2003 to 2004 latex agglutination (Slidex Rotavirus, Biomerieux, France) and since 2005 an immunochromatographic assay for the combined detection of rotavirus and adenovirus (Vikia Rota-Adeno, Biomerieux, France). This study was submitted and approved by the Internal Ethics Review Board of Albert Einstein Hospital.

A total of 3,768 stool samples were received during the study period, $55.2 \%$ were from males and $44.8 \%$ from females. Specimens were obtained from 1,350 hospitalized patients and 2,481 from outpatients. Age range was from one month to 89 years, median 12 years and in 2006 (53.2\%) of all samples were from children, age 0 to 10 years.

\section{Results}

Rotavirus was detected in 755 (20\%) from 3,768 samples, with $55.6 \%$ of cases in male patients. Of these positive cases, $352(46.6 \%)$ were from hospitalized patients. Positive samples were found in $26.1 \%$ of hospitalized patients and $16.7 \%$ of outpatients. Pediatric samples (0 to 10 years) comprised 856 samples of 1,350 total hospitalized patients (63.4\%). The setting with major prevalence of rotavirus infection was pediatrics $(35.6 \%)$.

Temporal distribution and positive tests incidence are shown in Figure 1. Positive tests were $19.8 \%$ in 2003, $21.7 \%$ in 2004 and $18.7 \%$ in 2005 . Positive tests were more frequent detected in July 2003 (39.5\%), August 2004 (39.2\%), and June $2005(35.7 \%)$.

Five hundred and seven positive tests were found in samples from children below five years old $(67.1 \%$ of all positive tests). Prevalence of $30 \%$ was found between six months and five years (Figure 2). In addition, all other age groups had a positive rate of at least $10 \%$. 
Figure 1. Monthly distribution of samples with positive and negative results during study period

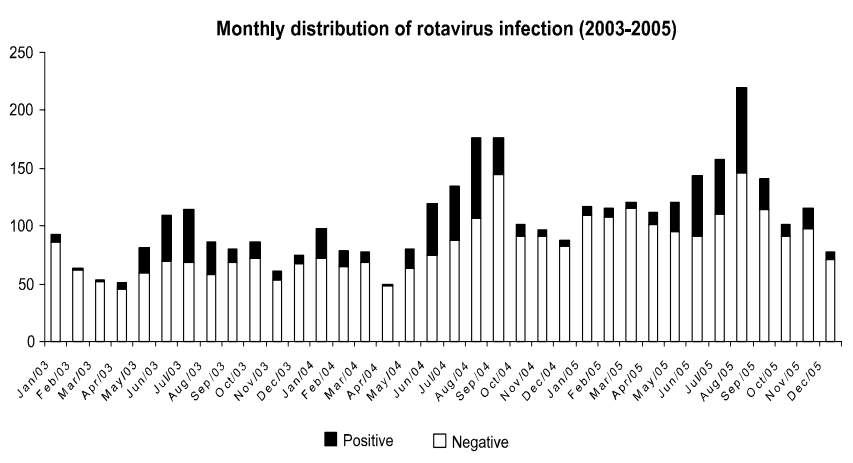

Figure 2. Rotavirus infections distribution by age (months and years)

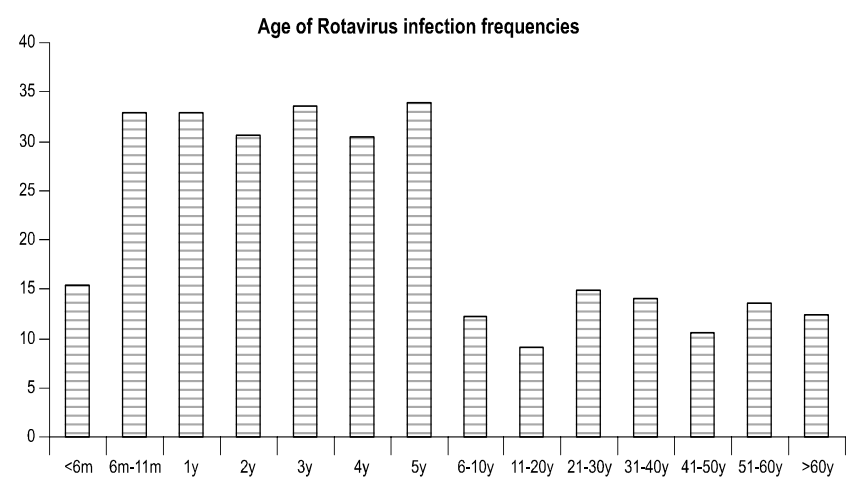

\section{Discussion}

Rotavirus infection rate varies with the population studied and with temporal, seasonal patterns. In this study, of all ordered rotavirus tests in stool samples, rotavirus antigen was found in $20 \%$ of 3,768 samples. Studies done in Brazil have shown prevalence rates of $11 \%$ to $42 \%$ in different cities [4].

Hospitalized patients had higher prevalence of positive tests than outpatients (26.5\% vs. $16.7 \%)$. This difference was higher in other studies done in Brazil: Silva et al. [10] found ten fold higher incidence of positive tests in hospitalized patients versus outpatients (32.6\% vs. 3.4\%) and Cardoso et al. [11] found a prevalence rate 14.7 times higher in hospitalized patients versus outpatients (23.8\% vs. $1.6 \%)$. Our hospital has an active program of infection control, based on alcohol gel use in hand washing and has a lower work load for health care workers compared with other hospitals in Brazil, and this can explain this difference. In all years studied we did not have hospital-acquired rotavirus infections in our hospital.

Pediatrics was the main setting where positive tests were found, confirming the importance of rotavirus infection as a cause of gastroenteritis leading to hospitalization in Brazil
[12]. Carneiro et al. [13] demonstrated that $39 \%$ of rotavirusassociated gastroenteritis seen at the Emergency room had to be admitted to the hospital for treatment. These data reinforce the implication described of rotavirus infections with more severe cases of childhood gastroenteritis [3].

Positive samples were detected through all three years of the study. Peak incidence of positive tests was between May and August, the winter months. This agrees with other studies done in temperate climate regions [14] and Brazilian studies done in Southern, Southeastern and Central-West Brazil $[10,15,16]$. Temporal occurrence of diarrhea admissions for children and deaths from diarrhea in Brazil occur in winter, suggesting the importance of rotavirus infection as cause of gastroenteritis in this patient population [17].

Frequencies over $30 \%$ of rotavirus infections were found between six months and five years of age, corresponding to $67 \%$ of all positive cases. Various studies in Brazilian populations have indicated that about $2 / 3$ of rotavirus infections occur in children under two years of age $[4,10,15,16]$. In our study, we found only $44.5 \%$ of positive cases in this age group (data not shown). In contrast, $33 \%$ of positive samples were from persons over six years old and $10 \%$ positive rates, at least, were found in all adults. A study similar to ours, done in a private hospital in Ponta Grossa, Paraná state, showed that $22.5 \%$ of all rotavirus infections were in persons aged more than six years [9]. Carmona et al. showed [18] 7.1\% positive rates of rotavirus detection in adults and elderly patients, and similar results were found in Paraguay [19]. Rates for rotavirus infection seem to be higher in South American adults than in developed countries, where they are between 2 to $5 \%$ [2].

Our data suggest that, in our patient population, adults do have rotavirus infection. This probably is not so well known in other patient populations because rotavirus rapid test is not available or because those tests are not done in adults with diarrhea. Adults have a wide variety of symptoms when they acquire rotavirus gastroenteritis, and patients with immune system deficiencies can have severe manifestations of this disease [20,21]. Anderson and Weber [2] described that adults with contact to children have a particular high risk of acquiring rotavirus gastroenteritis, rates going from 33 to $55 \%$ of adults that care for children at home with rotavirus gastroenteritis. Adults, like children, can have hospital-acquired rotavirus gastroenteritis and should be identified for isolation procedures for preventing this condition, as well as preventing antibiotic misuse.

Albert Einstein Hospital care for a different socioeconomic stratum than São Paulo population as a whole, but our findings probably can be extended to the city. Rotavirus infection was very frequent among population studied, with highest prevalence in young children hospitalized, but with also high frequency in outpatient adults. Clinicians caring for children and adults with gastroenteritis need to consider rotavirus in the differential diagnosis. 


\section{References}

1. Parashar U.D., Bresee J.S., Gentsch J.R., Glass R.I. Rotavirus. Emerg Infect Dis 1998;4:561-70.

2. Anderson E.J., Weber S.G. Rotavirus infection in adults. Lancet Infect Dis 2004;4:91-9.

3. Parashar U.D., Gibson C.J., Bresee J.S., Glass R.I. Rotavirus and severe childhood diarrhea. Emerg Infect Dis 2006;12:304-6.

4. Linhares A.C. Epidemiologia das infecções por rotavírus no Brasil e os desafios para seu controle. Cad Saúde Pública 2000;16:629-46.

5. Da Silva L.J. Rotavirus vaccine, a maturity symbol of immunization in Brazil. Pediatria 2005;27:221-2.

6. Caul E.O., Ashley C.R., Darville J.M., Bridger J.C. Group C rotavirus associated with fatal enteritis in a family outbreak. J Med Virol 1990; $30: 201-5$

7. Castro L., Rodrigues D.P., Flauzino R., Moura M., Leite J.P. An outbreak of diarrhoea associated with rotavirus serotype 1 in a day care nursery in Rio de Janeiro, Brazil. Mem Inst Oswaldo Cruz 1994;89:5-9.

8. Timenetsky M.C., Gouvea V., Santos N., et al. Outbreak of severe gastroenteritis in adults and children associated with type G2 rotavirus. J Diarrhoeal Dis Res 1996;14:71-4.

9. Pietruchinski E., Benati F., Lauretti F., et al. Rotavirus diarrhea in children and adults in a southern city of Brazil in 2003: distribution of G/P types and finding of a rare G12 strain. J Med Virol 2006; 78:1241-9.

10. Silva M.L.R., Naveca F.G., Carvalho I.P. Epidemiological aspects of rotavirus infections in Minas Gerais, Brazil. Braz J Infect Dis 2001;5:215-22.

11. Cardoso D.D.P., Soares C.M.A., Souza M.B.L.D., et al. Epidemiological features of rotavirus infection in Goiânia, Goiás, Brazil, from 1986 to 2000. Mem Inst Oswaldo Cruz 2003;98:25-9.
12. Carmona R.C., Timenetsky M.C., Silva F.F., Granato C.F. Characterization of rotavirus strains from hospitalized and outpatient children with acute diarrhea in Sao Paulo, Brazil. J Med Virol 2004;74:166-72.

13. Carneiro N.B., Diniz-Santos D.R., Fagundes S.Q., et al. Clinical and epidemiological aspects of children hospitalized with severe rotavirus-associated gastroenteritis in Salvador, BA, Brazil. Braz J Infect Dis 2005;9:525-8.

14. Cook S.M., Glass R.I., Le Baron C.W., Ho M. Global seasonality of rotavirus infection. Bull WHO 1990;68:171-7.

15. Costa P.S.S., Cardoso D.D.P., Grisi S.J.F.E., et al. Rotavirus A infections and reinfections: genotyping and vaccine implications. J Pediatr (Rio de J.) 2004;80:119-22.

16. Linhares A.C. Rotavirus Infection in Brazil: Epidemiology, Immunity, and Potential Vaccination. Braz J Infect Dis 1997; $1: 284-93$

17. Kale P.L., Fernandes C., Nobre F.F. Temporal pattern of diarrhea hospitalizations and deaths in children, 1995 to 1998, Brazil. Rev Saude Publica 2004;38:30-7.

18. Carmona R.C.C., Timenetsky M.C.S., Morillo S.G., Richtzenhain L.J. Human rotavirus serotype G9, São Paulo, Brazil, 19962003. Emerg Infect Dis 2006;12:963-8.

19. Martinez M., Farina N., Rodriguez M., et al. Incidence of rotavirus in adults with diarrhea in Asunción, Paraguay. Rev Argent Microbiol 2005;37:99-101.

20. Thomas P.D., Pollok R.C., Gazzard B.G. Enteric viral infections as a cause of diarrhea in the acquired immunodeficiency syndrome. HIV Med 1999;1:19-24.

21. Mori I., Matsumoto K., Sugimoto K., et al. Prolonged shedding of rotavirus in a geriatric inpatient. J Med Virol 2002;67:613-5. 